Research Article

\title{
Robust Fault Diagnosis Algorithm for a Class of Nonlinear Systems
}

\author{
Hai-gang Xu, Yue-feng Liao, and Xiao Han \\ Henan Mechanical and Electrical Engineering College, Xinxiang 453002, China \\ Correspondence should be addressed to Xiao Han; 1400651671@qq.com
}

Received 2 March 2015; Accepted 7 October 2015

Academic Editor: James Lam

Copyright (C) 2015 Hai-gang Xu et al. This is an open access article distributed under the Creative Commons Attribution License, which permits unrestricted use, distribution, and reproduction in any medium, provided the original work is properly cited.

\begin{abstract}
A kind of robust fault diagnosis algorithm to Lipschitz nonlinear system is proposed. The novel disturbances constraint condition of the nonlinear system is derived by group algebra method, and the novel constraint condition can meet the system stability performance. Besides, the defined robust performance index of fault diagnosis observer guarantees the robust. Finally, the effectiveness of the algorithm proposed is proved in the simulations.
\end{abstract}

\section{Introduction}

The complicated systems as aircraft, missile systems, and control system [1-6] easily show faults; how to diagnose the fault is a difficult problem to handle. When the systems show fault and you cannot isolate it, the systems will be collapsed. Therefore, the fault diagnosis and fault tolerant technology are meaningful to enhance the system performances. And fault diagnosis technology is foundation of the fault tolerance; in another way, fault tolerance is realized by the fault estimations information. The original control laws will be regulated by the fault estimation information, so the system reliability will be improved, particularly the missile control system. In this paper, we aim at the missile attitude control system. Robust fault diagnosis methods are useful ways to solve the systems with the disturbances and they are also proved effective in systems applications.

The robust fault diagnosis observer is designed based on unknown input observer theory in [7-9], and state estimation errors decouple from disturbances. Most of papers make assumptions that the disturbances constraint condition is known; this assumption limits the algorithm applications.

Dealing with the deficiency on assumption that the disturbance is norm bounded, a novel constraint condition for disturbance is designed. Besides, the defined robust performance index of fault diagnosis observer guarantees the robust. Furthermore, the threshold is designed in fault decision section.

\section{Problem Statement}

Consider the system uncertainty and unknown input disturbances, system state-space model:

$$
\begin{aligned}
\dot{\mathbf{x}}(t)= & (\mathbf{A}+\Delta \mathbf{A}) \mathbf{x}(t)+(\mathbf{B}+\Delta \mathbf{B}) \mathbf{u}(t) \\
& +\mathbf{h}(\mathbf{x}(t), \mathbf{u}(t))+\mathbf{g}_{0}(\mathbf{x}(t), \mathbf{u}(t), \mathbf{d}(t), t) \\
& +\mathbf{f}(t), \\
\mathbf{y}(t)= & \mathbf{C x}(t) .
\end{aligned}
$$

$\mathbf{E}_{1}, \mathbf{E}_{2}, \mathbf{F}_{1}$, and $\mathbf{F}_{2}$ are known matrix. $\Delta \mathbf{A}$ and $\Delta \mathbf{B}$ are model errors.

Therefore, we can get another form as

$$
\begin{aligned}
\dot{\mathbf{x}}(t)= & \mathbf{A x}(t)+\mathbf{B u}(t)+\mathbf{h}(\mathbf{x}(t), \mathbf{u}(t)) \\
& +\mathbf{g}(\mathbf{x}(t), \mathbf{u}(t), \mathbf{d}(t), t)+\mathbf{f}(t),
\end{aligned}
$$

where $\mathbf{x}(0)=\mathbf{x}_{0}$ is initial value, $\mathbf{x}(t) \in \mathbf{R}^{n}$ is state, $\mathbf{u}(t) \epsilon$ $\mathbf{R}^{p}$ and $\mathbf{y}(t) \in \mathbf{R}^{q}$ are system input and output, $\mathbf{g} \in \mathbf{R}^{n}$ is unknown input, nonlinear functions $\mathbf{g}$ and $\mathbf{h}$ are continuous 


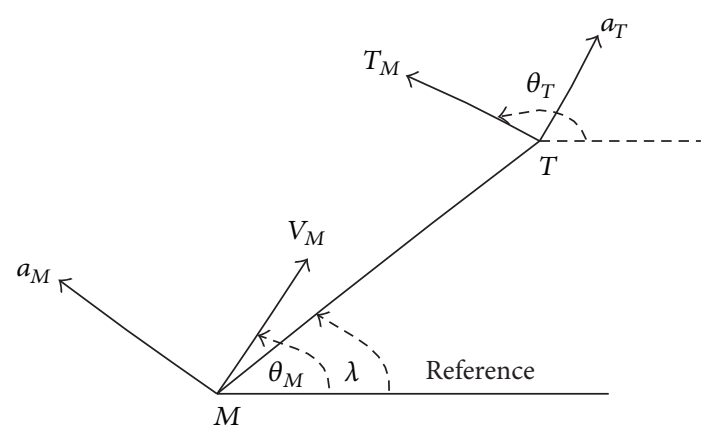

Figure 1: Missile and target geometry.

and differential, and $\mathbf{f}(t) \in \mathbf{R}^{n}$ is system fault. $\mathbf{A}, \mathbf{B}$, and $\mathbf{C}$ are known matrix.

The system state-space model can be obtained by Gronwall lemma [10-12]. Considering the complexity and particularity of missile control system, in the next two sections we perform the fault diagnosis algorithm from two aspects: stability and robust of the observer proposed.

Most of related papers assume that the constraint condition of external disturbances is norm bounded; however, a kind of nonlinear systems is unstable under the hypothetical constraint condition. Dealing with the limitations of disturbances constraint condition mentioned, a novel constraint condition of external disturbances is derived in Section 3; the systems hold stable under the condition proposed. Furthermore, the defined robust performance index of fault diagnosis observer guarantees the robust in Section 4.

The missile and target geometry are shown in Figures 1 and 2. The real system dynamics are described by the following differential equations:

$$
\begin{aligned}
m \frac{d v}{d t} & =p \cos \alpha-X-m g \sin \theta \\
m v \frac{d \theta}{d t} & =p \sin \alpha+Y-m g \cos \theta \\
J_{z} \frac{d \omega_{z}}{d t} & =M_{z} \\
\frac{d L}{d t} & =v \cos \theta \\
\frac{d H}{d t} & =v \sin \theta \\
\frac{d \vartheta}{d t} & =\omega_{z} \\
\alpha & =\vartheta-\theta .
\end{aligned}
$$

The system variables are Mach, longitudinal velocities and lateral velocities, control input, and so forth. The variables are demonstrated as follows.

\section{Stability Analysis}

Theorem 1. There exists $M \geq 1, \omega<0, t \geq 0$ to make nonlinear system (2) and (3) with external disturbances hold

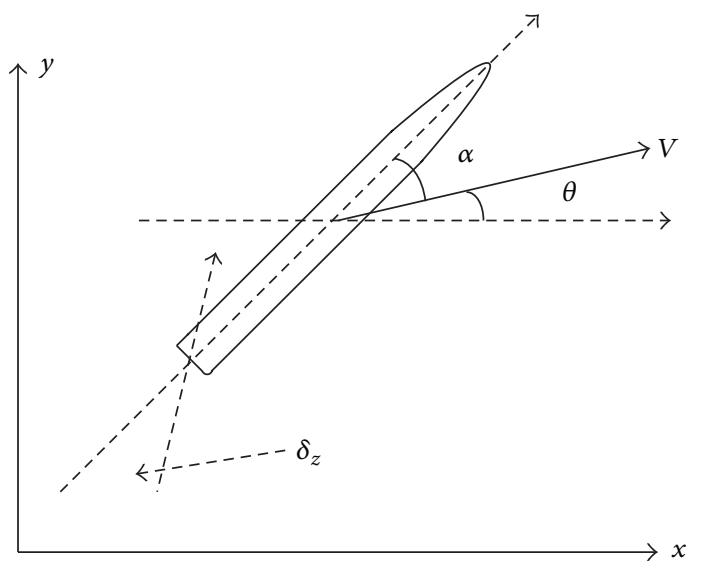

Figure 2: Air-frame axes.

stable, when the constraint condition of external disturbances is

$$
\|\mathbf{g}(\mathbf{x}(t), \mathbf{u}(t), \mathbf{d}(t), t)\|<\left(\beta_{1}(t)-\beta_{0}(t)\right)-\frac{\omega\|\mathbf{x}(\tau)\|}{M}
$$

and is defined as $\beta_{0}(t)=\|\mathbf{B u}(t)\|, \beta_{1}(t)=\|\mathbf{h}(\mathbf{x}(t), \mathbf{u}(t))\|$.

Proof. Matrix A is Hurwitz matrix and $\mathbf{A}$ can generate asymptotic convergence linear semigroup $\zeta_{t}$. Therefore, there exists $M \geq 1, \omega<0, t \geq 0$ such that the inequality holds:

$$
\left\|\zeta_{t}\right\| \leq M \exp (\omega t)
$$

Fault-free mode, state-space description of system (2) and (3) with external disturbances is

$$
\begin{aligned}
& \mathbf{x}(t)=\mathbf{x}(0)+\int_{0}^{t}[\mathbf{A x}(\tau)+\mathbf{B u}(\tau)+\mathbf{h}(\mathbf{x}(\tau), \mathbf{u}(\tau)) \\
& \quad+\mathbf{g}(\mathbf{x}(\tau), \mathbf{u}(\tau), \mathbf{d}(\tau), \tau)] d \tau
\end{aligned}
$$

Therefore, $\exists$ stable linear semigroup $\zeta_{t}$ makes (8) hold:

$$
\begin{aligned}
& \mathbf{x}(t)=\zeta_{t} \mathbf{x}(0)+\zeta_{t-\tau} \int_{0}^{t}[\mathbf{B u}(\tau)+\mathbf{h}(\mathbf{x}(\tau), \mathbf{u}(\tau)) \\
& \quad+\mathbf{g}(\mathbf{x}(\tau), \mathbf{u}(\tau), \mathbf{d}(\tau), \tau)] d \tau
\end{aligned}
$$

Apply the 2-norm to formula (8):

$$
\begin{aligned}
& \|\mathbf{x}(t)\|=\| \zeta_{t} \mathbf{x}(0)+\zeta_{t-\tau} \int_{0}^{t}[\mathbf{B u}(\tau)+\mathbf{h}(\mathbf{x}(\tau), \mathbf{u}(\tau)) \\
& \quad+\mathbf{g}(\mathbf{x}(\tau), \mathbf{u}(\tau), \mathbf{d}(\tau), \tau)] d \tau \|
\end{aligned}
$$

The simplification form of formula (9) is

$$
\begin{aligned}
\Xi= & \mathbf{B u}(\tau)+\mathbf{h}(\mathbf{x}(\tau), \mathbf{u}(\tau)) \\
& +\mathbf{g}(\mathbf{x}(\tau), \mathbf{u}(\tau), \mathbf{d}(\tau), \tau)
\end{aligned}
$$

Initial value of state is $\mathbf{x}_{0}=\mathbf{x}(0)$, we set $a=\|\mathbf{x}(0)\|$. From constraint of linear semigroup $\zeta_{t}$ in formula (6) and norm 
basic principle, the inequality constraint condition of formula (9) should be satisfied as follows:

$$
\|\mathbf{x}(t)\| \leq M a \exp (\omega t)+\int_{0}^{t}\|M \exp [\omega(t-\tau)] \Xi\| d \tau .
$$

Multiplied by $\exp (-\omega t)$ for two sides of formula (11):

$$
\begin{aligned}
\|\mathbf{x}(t)\| \exp (-\omega t) \leq & M a+\int_{0}^{t}\|M \exp (-\omega \tau) \Xi\| d \tau, \\
\|\mathbf{x}(t)\| \exp (-\omega t) \leq & M a \\
& +\int_{0}^{t} \frac{\|M \mathbf{x}(\tau) \exp (-\omega \tau) \Xi\|}{\|\mathbf{x}(\tau)\|} d \tau .
\end{aligned}
$$

By Gronwall lemma,

$$
\|\mathbf{x}(t)\| \leq M a \exp \left[\int_{0}^{t}\left(\omega+\frac{\|M \Xi\|}{\|\mathbf{x}(\tau)\|}\right) d \tau\right] .
$$

For simplification, we define as follows:

$$
\vartheta(t)=\int_{0}^{t}\left(\omega+\frac{\|M \Xi\|}{\|\mathbf{x}(\tau)\|}\right) d \tau .
$$

The system is stable for $\forall t \rightarrow+\infty$, $\lim _{t \rightarrow+\infty} \vartheta(t)<|\varepsilon|<$ $+\infty$ when there exists finite constant $|\varepsilon|<\infty$. There exists nonlinear semigroup $\pi_{t}$ such that state-space of system is described by the following from formula (9):

$$
\mathbf{x}(t)=\pi_{t} \mathbf{x}(0)
$$

From formulas (13) and (14):

$$
\|\mathbf{x}(t)\|=\left\|\pi_{t} \mathbf{x}(0)\right\| \leq M a \exp (\vartheta(t)) .
$$

Therefore, the nonlinear semigroup $\pi_{t}$ is stable when $\vartheta(t)<0$; in other words, system (2) and (3) with external disturbances is stable. As a result, the system holds stable when the following condition is satisfied:

$$
\|\Xi\|<-\frac{\omega\|\mathbf{x}(\tau)\|}{M} .
$$

Substitute the formula above into (10):

$$
\begin{aligned}
& \|\mathbf{B u}(t)+\mathbf{h}(\mathbf{x}(t), \mathbf{u}(t))+\mathbf{g}(\mathbf{x}(t), \mathbf{u}(t), \mathbf{d}(t), t)\| \\
& <-\frac{\omega\|\mathbf{x}(\tau)\|}{M} .
\end{aligned}
$$

And then

$$
\begin{aligned}
\|\mathbf{B u}(t)+\mathbf{h}(\mathbf{x}(t), \mathbf{u}(t))+\mathbf{g}(\mathbf{x}(t), \mathbf{u}(t), \mathbf{d}(t), t)\| \\
>\|\mathbf{B u}(t)\|-\|\mathbf{h}(\mathbf{x}(t), \mathbf{u}(t))\| \\
\quad+\|\mathbf{g}(\mathbf{x}(t), \mathbf{u}(t), \mathbf{d}(t), t)\|
\end{aligned}
$$

where, $\mathbf{u}(t)$ and $\mathbf{h}(\mathbf{x}(t), \mathbf{u}(t))$ are known. With definition $\beta_{0}(t)=\|\mathbf{B u}(t)\|, \beta_{1}(t)=\|\mathbf{h}(\mathbf{x}(t), \mathbf{u}(t))\|$.
Substitute into the formula above:

$$
\begin{aligned}
& \beta_{0}(t)-\beta_{1}(t)+\|\mathbf{g}(\mathbf{x}(t), \mathbf{u}(t), \mathbf{d}(t), t)\| \\
& \quad<-\frac{\omega\|\mathbf{x}(\tau)\|}{M}, \\
& \|\mathbf{g}(\mathbf{x}(t), \mathbf{u}(t), \mathbf{d}(t), t)\| \\
& \quad<\left(\beta_{1}(t)-\beta_{0}(t)\right)-\frac{\omega\|\mathbf{x}(\tau)\|}{M} .
\end{aligned}
$$

System (2) and (3) with external disturbances is stable when the constraint condition of external disturbances satisfies the form of (20). Furthermore, if there exists $\beta_{3} \in R^{+}$make $\left(\beta_{1}(t)-\beta_{0}(t)\right) \leq \beta_{3}\|\mathbf{x}(\tau)\|$, and therefore

$$
\|\mathbf{g}(\mathbf{x}(t), \mathbf{u}(t), \mathbf{d}(t), t)\|<\left(\beta_{3}-\frac{\omega}{M}\right)\|\mathbf{x}(\tau)\| .
$$

The constraint condition of external disturbances satisfies the inequation

$$
\frac{\|\mathbf{g}(\mathbf{x}(t), \mathbf{u}(t), \mathbf{d}(t), t)\|}{\|\mathbf{x}(\tau)\|}<\beta_{3}-\frac{\omega}{M} .
$$

The robustness performance index to external disturbances is defined as $\mathfrak{R}(\mathbf{A})=\omega / M$.

\section{Robust Fault Diagnosis Algorithm}

Theorem 2. The robust fault diagnosis observer (24) of system (2) and (3) with fault $\beta_{2}=\sup _{t \in[0, T]}\|\mathbf{f}(t)\|$ and external disturbances is asymptotic convergence. Therefore, the robust performance index of observer satisfies the inequality as follows:

$$
\begin{aligned}
-\mathfrak{R}(\mathbf{A}-\mathbf{G C})> & \lambda_{1} \\
& +\frac{\left(\|\mathbf{g}(\mathbf{x}(\tau), \mathbf{u}(\tau), \mathbf{d}(\tau), \tau)\|+\beta_{2}\right)}{\|\mathbf{e}(\tau)\|} .
\end{aligned}
$$

Proof. Construct robust fault diagnosis observer as follows for missile pitching motion control system (2) and (3) with fault and external disturbances:

$$
\begin{aligned}
\dot{\widehat{\mathbf{x}}}(t)= & \mathbf{A} \widehat{\mathbf{x}}(t)+\mathbf{B u}(t)+\mathbf{h}(\widehat{\mathbf{x}}(t), \mathbf{u}(t)) \\
& +\mathbf{G}[\mathbf{y}(t)-\widehat{\mathbf{y}}(t)] \\
\widehat{\mathbf{y}}(t)= & \mathbf{C} \widehat{\mathbf{x}}(t) .
\end{aligned}
$$

System states estimation errors and observer residuals are

$$
\begin{aligned}
& \mathbf{e}(t)=\mathbf{x}(t)-\dot{\widehat{\mathbf{x}}}(t), \\
& \mathbf{r}(t)=\mathbf{y}(t)-\widehat{\mathbf{y}}(t)
\end{aligned}
$$


And therefore,

$$
\begin{aligned}
\dot{\mathbf{e}}(t)= & \dot{\mathbf{x}}(t)-\dot{\hat{\mathbf{x}}}(t) \\
= & \mathbf{A x}(t)-\mathbf{A} \widehat{\mathbf{x}}(t)+\mathbf{h}(\mathbf{x}(t), \mathbf{u}(t)) \\
& -\mathbf{h}(\widehat{\mathbf{x}}(t), \mathbf{u}(t))-\mathbf{G}[\mathbf{C} \mathbf{x}(t)-\mathbf{C} \widehat{\mathbf{x}}(t)] \\
& +\mathbf{g}(\mathbf{x}(t), \mathbf{u}(t), \mathbf{d}(t), t)+\mathbf{f}(t), \\
\dot{\mathbf{e}}(t)= & (\mathbf{A}-\mathbf{G C}) \mathbf{e}(t)+\mathbf{h}(\mathbf{x}(t), \mathbf{u}(t)) \\
& -\mathbf{h}(\widehat{\mathbf{x}}(t), \mathbf{u}(t))+\mathbf{g}(\mathbf{x}(t), \mathbf{u}(t), \mathbf{d}(t), t) \\
& +\mathbf{f}(t), \\
\mathbf{r}(t)= & \mathbf{y}(t)-\widehat{\mathbf{y}}(t)=\mathbf{C e}(t) .
\end{aligned}
$$

The states estimation errors of system (26) are $\mathbf{e}_{0}=\mathbf{e}(0), b=$ $\|\mathbf{e}(0)\|$.

With definition: $\Psi=\mathbf{h}(\mathbf{x}(\tau), \mathbf{u}(\tau))-\mathbf{h}(\widehat{\mathbf{x}}(\tau), \mathbf{u}(\tau))+\mathbf{f}(t)+$ $\mathbf{g}(\mathbf{x}(\tau), \mathbf{u}(\tau), \mathbf{d}(\tau), \tau)$.

As a result, the states estimation errors are

$$
\begin{aligned}
\mathbf{e}(t)= & \exp [(\mathbf{A}-\mathbf{G C}) t] \mathbf{e}(0) \\
& +\int_{0}^{t} \exp [(\mathbf{A}-\mathbf{G C})(t-\tau)] \Psi d \tau .
\end{aligned}
$$

Apply the 2-norm to both sides of (27):

$$
\begin{aligned}
& \|\mathbf{e}(t)\|=\| \exp [(\mathbf{A}-\mathbf{G C}) t] \mathbf{e}(0) \\
& \quad+\int_{0}^{t} \exp [(\mathbf{A}-\mathbf{G C})(t-\tau)] \Psi d \tau \| .
\end{aligned}
$$

The system matrix A - GC is Hurwitz matrix when system (26) is stable; therefore, it can generate a stable linear semigroup $\zeta_{t}$.

Consequently, there exists $M \geq 1, \omega<0, t \geq 0$ such that $\left\|\zeta_{t}\right\| \leq M \exp (\omega t)$. Therefore, formula (28) fulfills the inequality as follows:

$$
\|\mathbf{e}(t)\| \leq M b \exp (\omega t)+\int_{0}^{t}\|M \exp [\omega(t-\tau)] \Psi\| d \tau
$$

Multiplied by $\exp (-\omega t)$ for formula (29):

$$
\begin{aligned}
\|\mathbf{e}(t)\| \exp (-\omega t) \leq & M b+\int_{0}^{t}\|M \exp (-\omega \tau) \Psi\| d \tau, \\
\|\mathbf{e}(t)\| \exp (-\omega t) \leq & M b \\
& +\int_{0}^{t} \frac{\|M \mathbf{e}(\tau) \exp (-\omega \tau) \Psi\|}{\|\mathbf{e}(\tau)\|} d \tau .
\end{aligned}
$$

Generally, the fault injected into the missile pitching motion control system is $\beta_{2}=\sup _{t \in[0, T]}\|\mathbf{f}(t)\|$ :

$$
\begin{aligned}
\|\Psi\| \leq & \|\mathbf{h}(\mathbf{x}(\tau), \mathbf{u}(\tau))-\mathbf{h}(\widehat{\mathbf{x}}(\tau), \mathbf{u}(\tau))\|+\|\mathbf{f}(t)\| \\
& +\|\mathbf{g}(\mathbf{x}(\tau), \mathbf{u}(\tau), \mathbf{d}(\tau), \tau)\| \\
\leq & \lambda_{1}\|\mathbf{e}(\tau)\|+\beta_{2} \\
& +\|\mathbf{g}(\mathbf{x}(\tau), \mathbf{u}(\tau), \mathbf{d}(\tau), \tau)\|, \\
\|\mathbf{e}(t)\| \leq & M b \exp \left\{\int_{0}^{t}\left[\omega+\frac{M\|\Psi\|}{\|\mathbf{e}(\tau)\|}\right] d \tau\right\} .
\end{aligned}
$$

Consequently, the fault diagnosis observer (24) is asymptotic convergence when the following formula holds:

$$
\begin{aligned}
\omega+\frac{M\|\Psi\|}{\|\mathbf{e}(\tau)\|} & <0, \\
-\frac{\omega}{M} & >\lambda_{1}+\frac{\left(\|\mathbf{g}(\mathbf{x}(\tau), \mathbf{u}(\tau), \mathbf{d}(\tau), \tau)\|+\beta_{2}\right)}{\|\mathbf{e}(\tau)\|} .
\end{aligned}
$$

The performance index $\Re(A-\mathbf{~ G C})$ of observer (24) satisfies the following constraint condition from stability theory:

$$
\begin{aligned}
-\Re(\mathbf{A}-\mathbf{G C})> & \lambda_{1} \\
& +\frac{\left(\|\mathbf{g}(\mathbf{x}(\tau), \mathbf{u}(\tau), \mathbf{d}(\tau), \tau)\|+\beta_{2}\right)}{\|\mathbf{e}(\tau)\|} .
\end{aligned}
$$

Consequently, the fault diagnosis observer is asymptotic convergence when formula (33) holds. And then, fault diagnosis observer can be realized by robust performance index proposed. Consider

$$
\begin{aligned}
\lambda_{i} & {\left[(\mathbf{A}-\mathbf{G C})+(\mathbf{A}-\mathbf{G C})^{T}\right] } \\
& <-2\left\{\lambda_{1}+\frac{\left(\|\mathbf{g}(\mathbf{x}(\tau), \mathbf{u}(\tau), \mathbf{d}(\tau), \tau)\|+\beta_{2}\right)}{\|\mathbf{e}(\tau)\|}\right\},
\end{aligned}
$$

where $\lambda_{\max }(\cdot)$ and $\lambda_{i}(\cdot)$ represent the maximum and arbitrary eigenvalue for matrix (.); the gain matrix $\mathbf{G}$ of the observer can be solved by pole assignment when the robust performance index $\mathfrak{R}(\mathbf{A}-\mathbf{G C})$ is given.

\section{Adaptive Threshold Design}

Usually, compare the residuals with threshold to diagnose fault.

The states estimation errors are

$$
\begin{aligned}
& \mathbf{e}(t)=\mathbf{e}(0)+\int_{0}^{t}[\mathbf{h}(\mathbf{x}(\tau), \mathbf{u}(\tau))-\mathbf{h}(\widehat{\mathbf{x}}(\tau), \mathbf{u}(\tau)) \\
& \quad+\mathbf{g}(\mathbf{x}(\tau), \mathbf{u}(\tau), \mathbf{d}(\tau), \tau)] d \tau
\end{aligned}
$$

Therefore,

$$
\begin{aligned}
\mathbf{r}(t) & =\mathbf{C e}(0)+\mathbf{C} \int_{0}^{t}[\mathbf{h}(\mathbf{x}(\tau), \mathbf{u}(\tau)) \\
& -\mathbf{h}(\widehat{\mathbf{x}}(\tau), \mathbf{u}(\tau))+\mathbf{g}(\mathbf{x}(\tau), \mathbf{u}(\tau), \mathbf{d}(\tau), \tau)] d \tau .
\end{aligned}
$$


Apply the 2-norm for formula (36):

$$
\begin{aligned}
& \|\mathbf{r}(t)\|=\| \mathbf{C e}(0)+\mathbf{C} \int_{0}^{t}[\mathbf{h}(\mathbf{x}(\tau), \mathbf{u}(\tau)) \\
& -\mathbf{h}(\widehat{\mathbf{x}}(\tau), \mathbf{u}(\tau))+\mathbf{g}(\mathbf{x}(\tau), \mathbf{u}(\tau), \mathbf{d}(\tau), \tau)] d \tau \|
\end{aligned}
$$

with definition $b_{c}=\|\mathrm{C}\|$ and

$$
\delta(\mathbf{h})=\mathbf{h}(\mathbf{x}(\tau), \mathbf{u}(\tau))-\mathbf{h}(\widehat{\mathbf{x}}(\tau), \mathbf{u}(\tau)) .
$$

Therefore,

$$
\begin{aligned}
& \|\mathbf{r}(t)\| \\
& \quad \leq b b_{c} \\
& \quad+b_{c}\left\|\int_{0}^{t}[\delta(\mathbf{h})+\mathbf{g}(\mathbf{x}(\tau), \mathbf{u}(\tau), \mathbf{d}(\tau), \tau)] d \tau\right\| .
\end{aligned}
$$

We can get from (38)

$$
\begin{aligned}
\|\delta(\mathbf{h})\| & \leq \lambda_{1}\|\mathbf{x}(\tau)-\widehat{\mathbf{x}}(\tau)\|=\lambda_{1}\|\mathbf{e}(\tau)\| \\
& =\frac{\lambda_{1}}{b_{c}}\|\mathbf{r}(\tau)\| .
\end{aligned}
$$

As a result,

$$
\begin{aligned}
& \|\mathbf{r}(t)\| \leq b b_{c} \\
& \quad+\int_{0}^{t}\left[\lambda_{1}\|\mathbf{r}(\tau)\|+b_{c}\|\mathbf{g}(\mathbf{x}(\tau), \mathbf{u}(\tau), \mathbf{d}(\tau), \tau)\|\right] d \tau .
\end{aligned}
$$

We can get from (41)

$$
\begin{aligned}
\|\mathbf{r}(t)\| \leq & b b_{c}+\int_{0}^{t} \lambda_{1} b_{c}\|\mathbf{e}(\tau)\| d \tau \\
& +\int_{0}^{t} b_{c}\left[\beta_{1}(\tau)-\beta_{0}(\tau)-\frac{\omega\|\mathbf{x}(\tau)\|}{M}\right] d \tau,
\end{aligned}
$$

where maximum tolerant values of estimation errors $\mathbf{e}_{\max }$ and the system stable value $\mathbf{x}_{\text {sta }}$ are known:

$$
\begin{aligned}
\mathbf{e}_{\max } & =\left\|\mathbf{e}_{\max }(t)\right\|, \\
\lim _{t \rightarrow T}\|\mathbf{x}(t)\| & \longrightarrow \mathbf{x}_{\text {sta }} .
\end{aligned}
$$

It can be obtained from (42) that

$$
\begin{aligned}
\|\mathbf{r}(t)\| \leq & b b_{c}+\lambda_{1} b_{c} \mathbf{e}_{\max } t \\
& +b_{c} \int_{0}^{t}\left[\beta_{1}(\tau)-\beta_{0}(\tau)-\frac{\omega x_{\max }}{M}\right] d \tau .
\end{aligned}
$$

As a result, the adaptive threshold of the fault diagnosis observer designed is

$$
\begin{aligned}
\mathbf{J}_{\text {th }}(\mathbf{r}(t))= & b b_{c}+\left(\lambda_{1} b_{c} \mathbf{e}_{\max }-\frac{\omega x_{\max }}{M}\right) t \\
& +b_{c} \int_{0}^{t}\left[\beta_{1}(\tau)-\beta_{0}(\tau)\right] d \tau .
\end{aligned}
$$

\section{Simulation}

In order to verify the effectiveness of the algorithm proposed, the following simulations are performed.

6.1. Simulation Parameters. The differential equations of missile pitching motion control system are represented as follows [6].

The pitching moment: $M_{z} \approx \varphi_{1}(m a, \alpha)+\varphi_{2}(m a, \alpha) \delta_{z}+$ $\varphi_{3}\left(\alpha, \omega_{z}, v\right)$. The missile aerodynamic parameters are as follows: $X(m a, \alpha), Y(m a, \alpha), \varphi_{1}(m a, \alpha), \varphi_{2}(m a, \alpha)$, and $\varphi_{3}\left(\alpha, \omega_{z}, v\right)$. The force situation of missile with different flight states is different.

The missile empty weight is $230 \mathrm{~kg}, z$-axis rotational inertia is $J_{z}=247.26 \mathrm{~kg} \cdot \mathrm{m}^{2}$, and generator impulse thrust of missile attitude control system is $p=2200 \mathrm{~N}$. The initial location of missile in inertial coordinates system is $x_{0}=$ $8530 \mathrm{~m}, y=11600 \mathrm{~m}$; missile initial velocity is $v=300 \mathrm{~m} / \mathrm{s}$, and trajectory pitching angle is $\theta=0.536 \mathrm{rad}$.

The system disturbances are $d_{i}(t)=0.01 \sin (t), i=$ $1,2, \ldots, 7$. The constraint condition of the external disturbances can be derived by Section 3 .

6.2. Performance Analysis for the Fault Diagnosis Algorithm. The supreme of missile external disturbances in the considered period under the Simulink condition from Theorem 1 is $\|\mathbf{g}\|_{\max }=9.69507 \times 10^{5}$. Therefore, not all of the disturbances can satisfy norm bounded constraint conditions and the prior constraint condition on external disturbances restricts the generality of fault diagnosis applications. The maximum value of estimation errors is defined as

$$
\mathbf{e}_{\max }=\left[\begin{array}{lllllll}
40 & 0.01 & 2 & 20 & 20 & 0.02 & 0.02
\end{array}\right]
$$

Therefore, $\|\mathbf{e}\|_{\max }=1.202 \times 10^{3}$. From Theorem 2,

$$
\lambda_{i}\left[(\mathbf{A}-\mathbf{G C})+(\mathbf{A}-\mathbf{G C})^{T}\right]<-806.5786 .
$$

The fault diagnosis observer designed is asymptotic convergence robust fault diagnosis observer when the poles are placed at the left plane of -806.5786 . It should be noticed that the gain matrix $\mathbf{G}$ is not unique because of the poles selected; in this paper, the poles are settled on

$$
\begin{aligned}
\mathbf{p}= & {[-850+5 \mathrm{i},-850-5 \mathrm{i},-880+6 \mathrm{i},-880-6 \mathrm{i},} \\
& -900+10 \mathrm{i},-900-10 \mathrm{i},-1000] .
\end{aligned}
$$

The gain matrix $\mathbf{G}$ of the observer can be get through the pole assignment.

As a result, the missile trajectory character is depicted in Figure 3 and substitute the gain matrix G into the observer (26) and then the residual effect is depicted in Figure 4 by Simulink. Without loss of generality, just take the 3rd channel residual of fault diagnosis as an example to research and assume the missile attitude control system.

Residual is asymptotic convergence and therefore it has the robustness to disturbances from Figure 4 . The good convergence of residual illustrates that the algorithm proposed is effective. Furthermore, the smooth residual curve also 


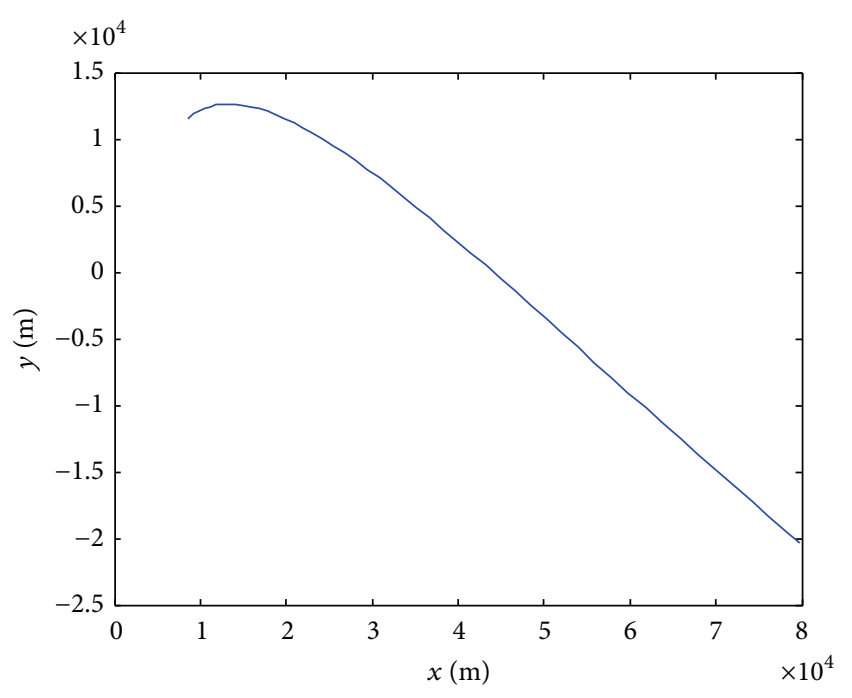

FIgURE 3: The missile trajectory character.

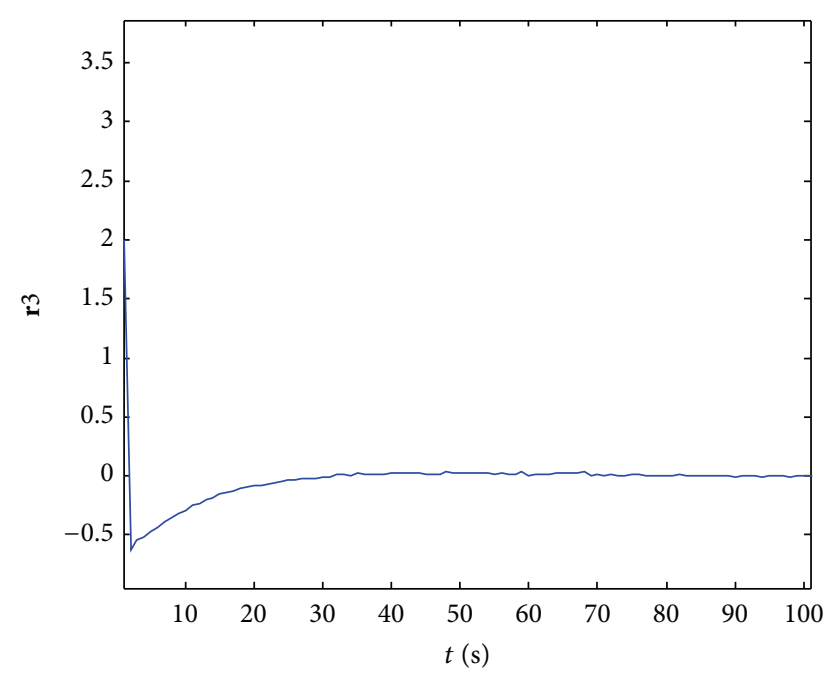

FIgURE 4: The 3rd channel residual of the observer.

illustrates that the disturbances constraint condition which can satisfy the system stability is reasonable and the defined robust performance index is practicable.

\section{Conclusion}

In this paper, novel disturbances constraint condition is derived to improve the limitation that external disturbance is norm bounded. And then, the novel constraint condition can meet the system stability. Besides, the defined robust performance index of fault diagnosis observer guarantees the robust. In decision-making unit, adaptive threshold is designed. Finally, simulation results show the effectiveness of the algorithm proposed.

\section{Conflict of Interests}

The authors declare that there is no conflict of interests regarding the publication of this paper.

\section{References}

[1] H. Buschek, "Design and flight test of a robust autopilot for the IRIS-T air-to-air missile," Control Engineering Practice, vol. 11, no. 5, pp. 551-558, 2003.

[2] Q. Hu, B. Xiao, and M. I. Friswell, "Fault tolerant control with $\mathrm{H}_{\infty}$ performance for attitude tracking of flexible spacecraft," IET Control Theory and Applications, vol. 6, no. 10, pp. 13881399, 2012.

[3] S. Chaib, D. Boutat, A. Banali, and F. Kratz, "Invertibility of switched nonlinear systems. Application to missile faults reconstruction," in Proceedings of the 46th IEEE Conference on Decision and Control (CDC '07), pp. 3239-3244, IEEE, New Orleans, La, USA, December 2007.

[4] M. A. Wael and Q. Quan, "Robust hybrid control for ballistic missile longitudinal autopilot," Chinese Journal of Aeronautics, vol. 24, no. 6, pp. 777-788, 2011.

[5] A. Tsourdos and B. A. White, "Adaptive flight control design for nonlinear missile," Control Engineering Practice, vol. 13, no. 3, pp. 373-382, 2005.

[6] G. M. Siouris, Missile Guidance and Control Systems, Springer, New York, NY, USA, 2004.

[7] J. Zarei and J. Poshtan, "Sensor fault detection and diagnosis of a process using unknown input observer," Mathematical and Computational Applications, vol. 16, no. 1, pp. 31-42, 2011.

[8] C. Hajiyev and F. Caliskan, "Sensor and control surface/actuator failure detection and isolation applied to F-16 flight dynamic," Aircraft Engineering and Aerospace Technology, vol. 77, no. 2, pp. 152-160, 2005.

[9] H. R. Karimi, M. Zapateiro, and N. Luo, "A linear matrix inequality approach to robust fault detection filter design of linear systems with mixed time-varying delays and nonlinear perturbations," Journal of the Franklin Institute, vol. 347, no. 6, pp. 957-973, 2010.

[10] A. Pazy, Semi-Groups of Linear Operators and Applications to Partial Differential Equations, Springer, New York, NY, USA, 1983.

[11] P. A. Ioannou and J. Sun, Robust Adaptive Control, Prentice Hall, New York, NY, USA, 1996.

[12] H.-M. Qian, Z.-D. Fu, J.-B. Li, and L.-L. Yu, "Robust fault diagnosis algorithm for a class of Lipschitz system with unknown exogenous disturbances," Measurement, vol. 46, no. 8, pp. 2324$2334,2013$. 

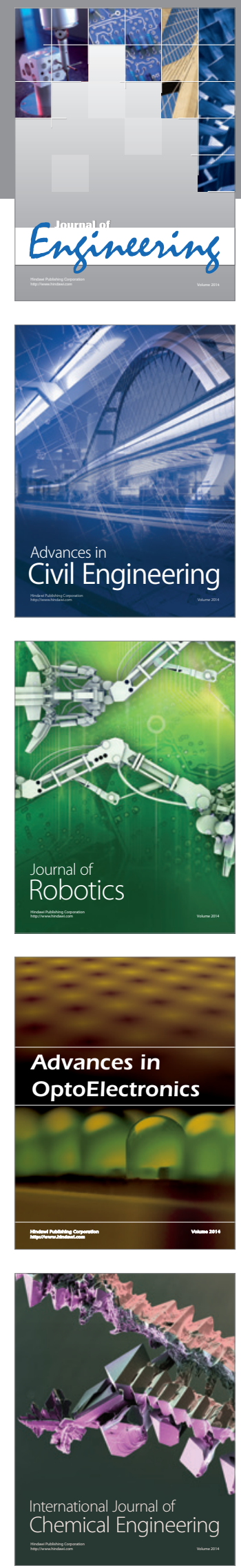

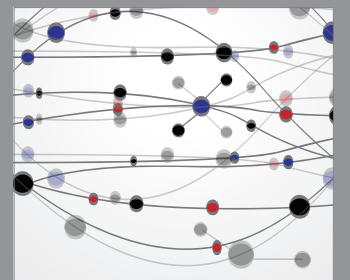

The Scientific World Journal
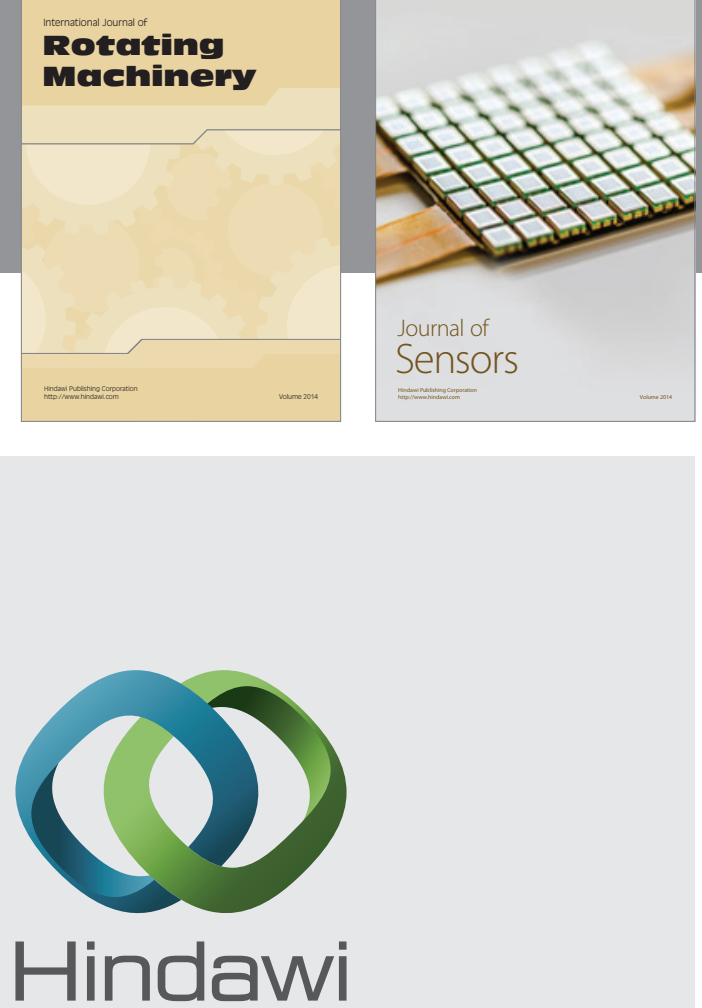

Submit your manuscripts at http://www.hindawi.com
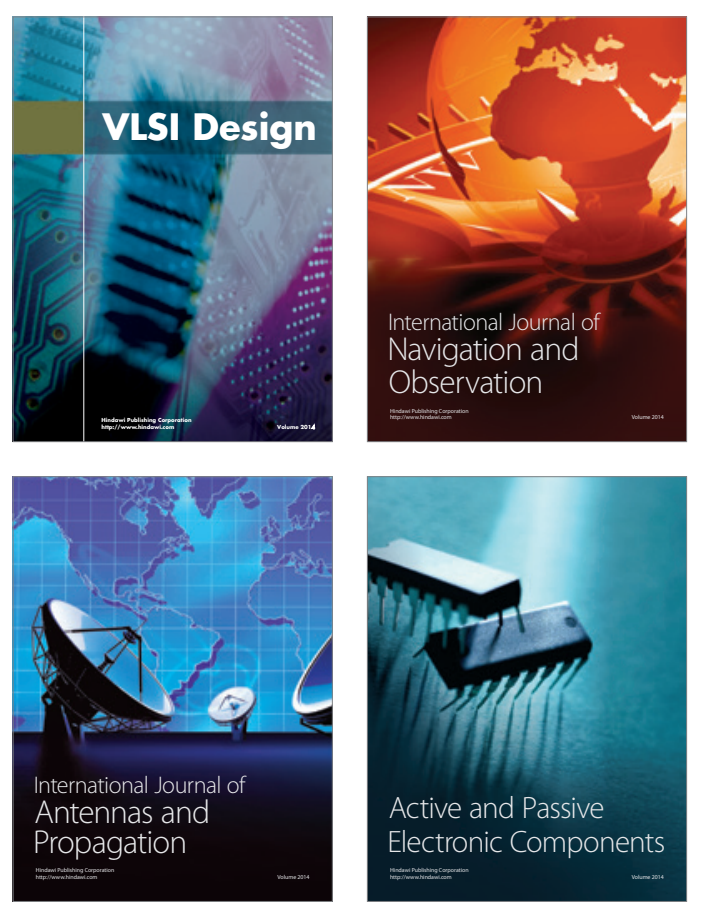
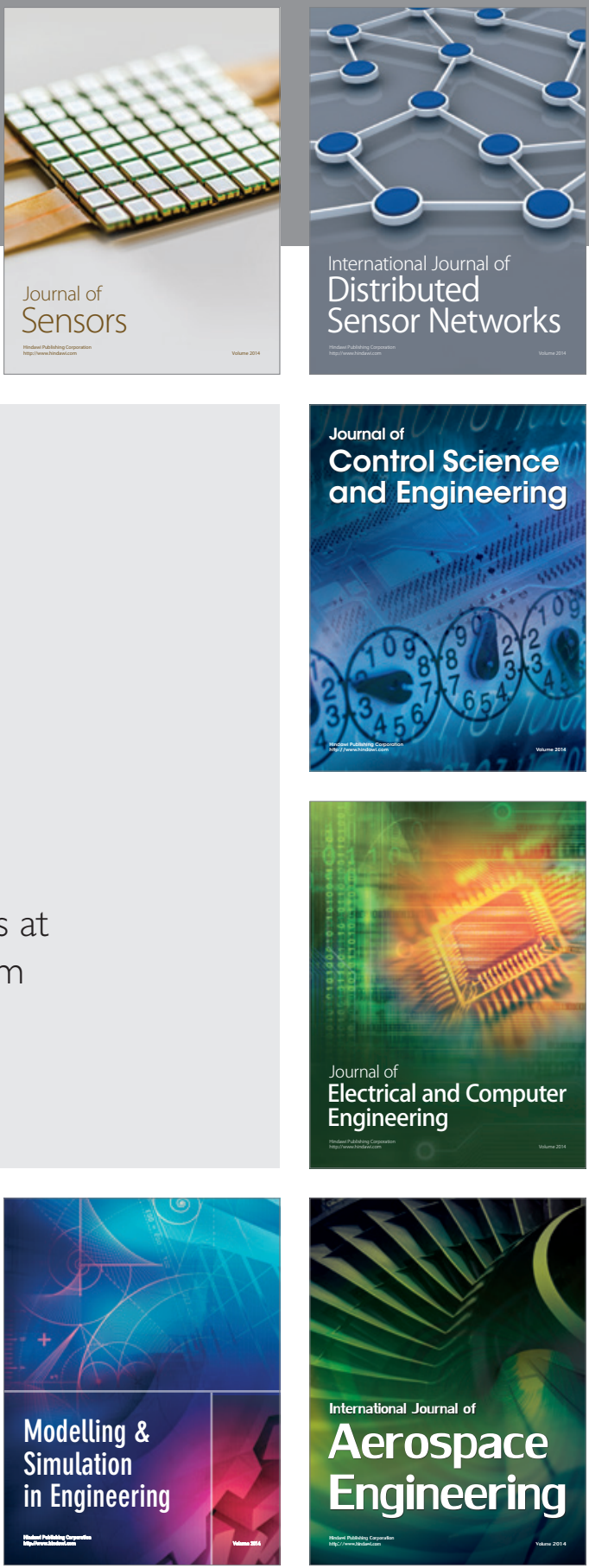

Journal of

Control Science

and Engineering
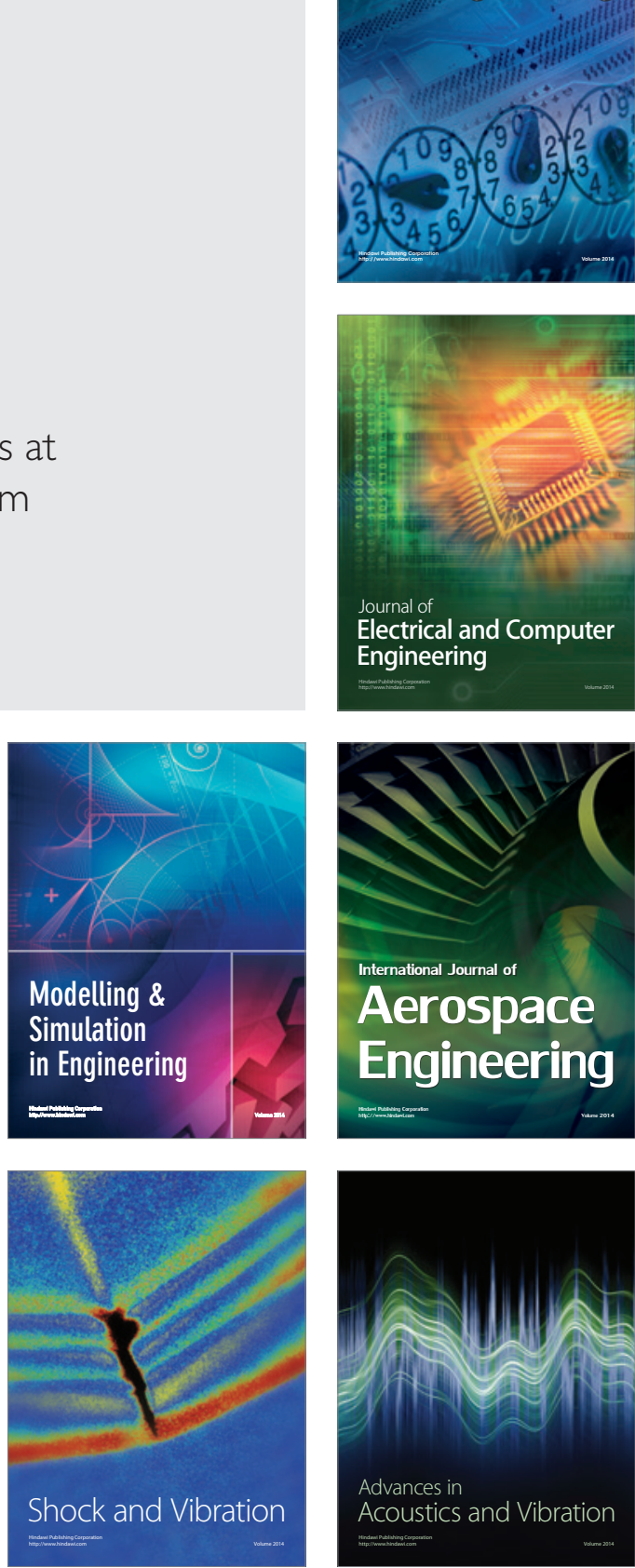